\title{
Decay properties of the X-ray afterglows of gamma-ray bursts
}

\author{
B. Gendre ${ }^{1}$ and M. Boër ${ }^{2}$ \\ 1 Istituto di Astrofisica Spaziale e Fisica Cosmica, Via Fosso del Cavaliere 100, 00133 Roma, Italy \\ e-mail: gendre@rm.iasf.cnr.it \\ 2 Observatoire de Haute-Provence, 04870 St. Michel l'Observatoire, France \\ e-mail: Michel.Boer@oamp.fr
}

Received 20 September 2004 / Accepted 2 November 2004

\begin{abstract}
We present a set of seventeen Gamma-Ray Bursts (GRBs) with known redshifts and X-ray afterglow emission. We apply cosmological corrections in order to compare their fluxes normalized at a redshift of 1 . Two classes of GRB can be defined using their X-ray afterglow light curves. We show that the brightest afterglows seem to decay faster than the dimmer ones. We also point out evidence for a possible flux limit of the X-ray afterglow depending on the time elapsed since the burst. We try to interpret these observations in the framework of the canonical fireball model of GRB afterglow emission.
\end{abstract}

Key words. gamma rays: bursts - X-rays: general

\section{Introduction}

Gamma-ray bursts (GRBs) are among the most enigmatic phenomena in the Universe. Since their discovery in the late 60's (Klebesadel et al. 1973) and until the BeppoSAX revolution, the observer faced a growing sample with no or few constrained properties. One of these properties, discovered using the the PHEBUS and BATSE observations, was that GRBs could be separated in two classes, according to their duration and spectral properties (Dezalay et al. 1996; Kouveliotou et al. 1993). Another step forward was made by the BeppoSAX satellite team, which succeeded in detecting a GRB X-ray afterglow (Costa et al. 1997). A new generation of automated telescopes such as TAROT (Boër et al. 1999) or ROTSE (Akerlof et al. 2000) was built in order to detect quickly the optical counterpart of GRBs.

To date, only long GRB afterglows were observed. Optical observations showed that GRBs are located at cosmological distances (Metzger et al. 1997). This made it possible to rule out some emission models, and favored the emergence of the fireball model (Rees \& Meszaros 1992; Meszaros \& Rees 1997; Panaitescu et al. 1998). In this model a blast wave propagates into a surrounding medium. The afterglow emission is described as synchrotron and inverse Compton emission of high energy electrons accelerated during the shock of an ultra-relativistic shell with the external medium, while the prompt emission is due to the internal shocks produced by shells of different Lorentz factors within the relativistic blast wave (see Piran 1999, for a review). In the first development of this model this medium was uniform, and the blast wave was isotropic. This case is referred to as the InterStellar Medium (ISM) model. Two refinements were made later. First, achromatic breaks in the light curves of some afterglows were explained by the non-isotropy (i.e. jet character) of the blast wave (e.g. Pian et al. 2001). This model without the isotropy assumption was called the "jet model" (Rhoads 1997; Sari et al. 1999). Second, in some case the optical afterglow light curves show bumps associated with type Ic supernovae (Reichart 1999). These and X-ray features (e.g. Piro et al. 1999; Reeves et al. 2002) show that long GRBs may be linked with the explosion of a massive star (hypernova; Meszaros 2001). If this is the case, the surrounding medium should not be uniform (Chevalier \& Li 2000): its density decreases with the square of the distance to the central engine, due to the wind from the progenitor of the GRB. This model is referred as the "wind model" (Dai \& Lu 1998; Meszaros et al. 1998; Panaitescu et al. 1998; Chevalier \& Li 1999). Each of these models is defined by a set of parameters, some of them referring to the microphysics ( $p, \epsilon_{e}, \epsilon_{B}$ ), others to the central engine $(E, \theta)$, and the remaining parameters depending on the surrounding medium ( $n$, absorption,...). They also lead to different spectral and temporal characteristics of the afterglows.

Because their brightness makes it possible to detect them at large cosmological distances, and because of the growing evidence that at least some long gamma-ray bursts are linked with type Ic supernovae, it is tempting to study whether some of their observational properties are reproduced from burst to burst. In this case, GRBs could be used to study the Universe, specifically the region of re-ionization. A first attempt was made by Boër \& Gendre (2000, hereafter Paper I): while looking for evidence for optical absorption around the burst, they discovered that GRB X-ray afterglows with known redshifts have a bimodal luminosity evolution: the faintest 
Table 1. Some data for the GRB sources we used in the present work. We indicate the observed flux one day after the burst. See definitions of groups I and II in the text.

\begin{tabular}{|c|c|c|c|c|c|c|c|c|}
\hline $\begin{array}{c}\text { Source } \\
\text { name }\end{array}$ & Group & $\begin{array}{c}\text { X-ray } \\
\text { satellite }\end{array}$ & Redshift & $\begin{array}{l}\text { Decay } \\
\text { index }\end{array}$ & $\begin{array}{l}\text { Spectral } \\
\text { index }\end{array}$ & $\begin{array}{c}\text { X-ray flux } \\
\text { @ } 1 \text { day } \\
\left(10^{-12} \mathrm{erg} \mathrm{cm}^{-2} \mathrm{~s}^{-1}\right)\end{array}$ & $\begin{array}{c}\text { Beaming } \\
\text { angle }^{a}\end{array}$ & Reference \\
\hline GRB 971214 & $\bar{I}$ & BeppoSAX & 3.42 & $1.6 \pm 0.1$ & $1.2 \pm 0.4$ & $0.23 \pm 0.05$ & $>0.1$ & 1,2 \\
\hline GRB 990123 & I & BeppoSAX & 1.60 & $1.44 \pm 0.11$ & $1.00 \pm 0.05$ & $1.8 \pm 0.4$ & 0.089 & 3 \\
\hline GRB 990510 & I & BepooSAX & 1.619 & $1.4 \pm 0.1$ & $1.2 \pm 0.2$ & $1.2 \pm 0.2$ & 0.054 & 4,5 \\
\hline GRB 991216 & I & RXTE, Chandra & 1.02 & $1.6 \pm 0.1$ & $0.8 \pm 0.5$ & $5.6 \pm 0.3$ & 0.051 & $6,7,8$ \\
\hline GRB 000926 & I & BeppoSAX, Chandra & 2.066 & $1.7 \pm 0.5$ & $0.7 \pm 0.2$ & - & 0.14 & 9 \\
\hline GRB 010222 & I & BeppoSAX, Chandra & 1.477 & $1.33 \pm 0.04$ & $1.01 \pm 0.06$ & $2.7 \pm 0.6$ & 0.08 & 9 \\
\hline GRB 970228 & II & BeppoSAX & 0.695 & $1.3 \pm 0.2$ & $0.8 \pm 0.3$ & $0.9 \pm 0.4$ & - & 1,10 \\
\hline GRB 970508 & II & BeppoSAX & 0.835 & $1.1 \pm 0.1$ & $1.1 \pm 0.3$ & $1.0 \pm 0.4$ & 0.391 & $1,11,12$ \\
\hline GRB 980613 & II & BeppoSAX & 1.096 & $1.1 \pm 0.2$ & - & $0.27 \pm 0.07$ & $>0.226$ & 1,13 \\
\hline GRB 980703 & II & BeppoSAX & 0.966 & $0.9 \pm 0.2$ & $1.8 \pm 0.4$ & $0.48 \pm 0.07$ & 0.2 & 14 \\
\hline GRB 000210 & II & BeppoSAX, Chandra & 0.846 & $1.38 \pm 0.03$ & $0.9 \pm 0.2$ & $0.21 \pm 0.06$ & - & 15 \\
\hline GRB 000214 & II & BeppoSAX & $0.37-0.47$ & $0.7 \pm 0.3$ & $1.2 \pm 0.5$ & $0.6 \pm 0.2$ & - & 9 \\
\hline GRB 011121 & II & BeppoSAX & 0.36 & $4_{-2}^{+3}$ & $2.4 \pm 0.4$ & $0.6 \pm 0.2$ & - & 9 \\
\hline GRB 011211 & II & XMM-Newton & 2.14 & $1.3 \pm 0.1$ & $1.2 \pm 0.1$ & $0.03 \pm 0.01$ & - & 15 \\
\hline GRB 030226 & II & Chandra & 1.98 & $2.7 \pm 1.6$ & $0.9 \pm 0.2$ & - & - & 15 \\
\hline GRB 030329 & II & RXTE, XMM-Newton & 0.168 & $0.9 \pm 0.3$ & $0.9 \pm 0.2$ & $14.3 \pm 2.9$ & - & 15,16 \\
\hline GRB 980425 & - & BeppoSAX & 0.0085 & $0.16 \pm 0.04$ & $1.0 \pm 0.3$ & $0.47 \pm 0.07$ & - & 17 \\
\hline
\end{tabular}

${ }^{a}$ Values extracted from Berger et al. (2003).

NB: The observations of GRB 000926 and GRB 030226 did not allow a meaningful extrapolation of the light curves. We thus cannot estimate the X-ray flux one day after the burst for these two events. The flux of GRB 000926 was $1.2 \times 10^{-13} \pm 0.1 \times 10^{-13} \mathrm{erg} \mathrm{s}^{-1} \mathrm{~cm}^{-2} 2.78 \mathrm{days}$ after the burst and that of GRB 030226 was $3.5 \times 10^{-14} \pm 0.2 \times 10^{-14} \mathrm{erg} \mathrm{s}^{-1} \mathrm{~cm}^{-2} 1.77$ days after the burst. The spectral index of GRB 980613 has never been reported, we assumed a value of 1 .

References: 1 Costa (1999); 2 Diercks et al. (1998); 3 Galama et al. (1999a); 4 Kuulkers et al. (2000); 5 Stanek et al. (1999); 6 Piro et al. (2000); 7 Halpern et al. (2000); 8 Frail et al. (2000); 9 De Pasquale et al. (2004); 10 Galama et al. (1997); 11 Pedersen et al. (1998); 12 Piro et al. (1998); 13 Djorgovski et al. (1998); 14 Vreeswijk et al. (1999); 15 This work; 16 Tiengo et al. (2003); 17 Pian et al. (2000).

GRB afterglows appear to decay more slowly than the brighter ones. They found that bright and faint X-ray afterglows were separated by one order of magnitude in flux one day after the burst. Frail et al. (2001) showed evidence that the energy released during the prompt emission of the GRB may be roughly constant. In this case the observed range in luminosity is due to beaming effects.

In this paper we reexamine our work presented in Paper I, using a larger sample of X-ray afterglows. At that time our sample was not large enough to draw strong conclusions (8 events only). Thanks to the Chandra and XMM-Newton X-ray observatories the sample has grown enough to look for a meaningful correlation. This paper is organized as follow. The data are presented in Sect. 2, together with the processing methods. We describe the normalization applied to the GRB afterglow light curves in Sect. 3. The results are given in Sect. 4 and discussed in Sect. 5, before the conclusions.

\section{The data}

Our sample is listed in Table 1. The first column refers to the source name, the third column to the satellites that we used to compute the X-ray afterglow light curves, while the fourth column gives the source redshift. We used only GRBs with known redshifts that exhibit an X-ray afterglow observed either by BeppoSAX, XMM-Newton or Chandra (ACIS imaging mode only). We included GRB 980425 despite its very uncommon properties (very low redshift, slow X-ray decay). For the BeppoSAX bursts we used either published results or data from De Pasquale et al. (2004). For the Chandra and XMM-Newton observations we reduced the data using the most up-to-date SAS and CIAO softwares (version 6.0 for the SAS, 3.2 for CIAO). Table 1 gives the main characteristics of each burst, in the observer frame (no corrections for distance or cosmological effects are included in the table).

We processed the data as follows: we first recalibrated the event lists, using the tasks emchain, epchain and acis_process_events; these event files were then filtered using the standard procedures depending on the instrument and satellite (we refer the reader to e.g. Gendre et al. 2003,for the details of these procedures); we looked for any period of high background and exclude any occurrence of it. We finally filtered the event files, keeping only events with energy between 0.3 and $10.0 \mathrm{keV}$. These filtered event lists were used to extract light curves and spectra of each afterglow. We used the XSPEC software (Arnaud 1996) to fit the spectra with a canonical power law model (taking into account any absorption needed to fit the data), and to derive a mean flux in the $2.0-10.0 \mathrm{keV}$ band. This makes it possible to calibrate the light curves in flux units. 
In the case of GRB 011211, observed by XMM-Newton, the telescope was re-pointed during the observation. Such a change in the satellite attitude is currently not fully supported by the SAS. We thus discarded the first $5 \mathrm{ks}$ of the observation, and we used only the data recorded after the re-pointing.

We also did not include the second XMM-Newton observation of GRB 030329, because of the large contamination by a source close to the afterglow position. For this burst we decided to use the results from Tiengo et al. (2003).

\section{The normalization of the X-ray afterglow light curves}

Because we decided to compare the fluxes of several X-ray afterglows of GRBs, we needed to normalize them. This normalization is similar to the one computed in Paper I. We have corrected the fluxes for distance, time dilatation, and energy losses due to the cosmological energy shift. To compute these corrections, we used a flat universe model, with an $\Omega_{\mathrm{m}}$ value of 0.3 .

The distance correction linearly depends on the value of $H_{0}$, the Hubble constant. Because this value is not well constrained, we normalized the flux to a common distance rather than using the luminosity. We normalized all X-ray light curves to a common distance corresponding to a redshift of $z=1$. These corrections do not take into account any beaming due to a possible jet. The distance used is based on the luminosity distance given by Penn (1999).

We corrected the cosmological energy shift as in Lamb \& Reichart (2000). This implies knowing the spectral slope of the afterglow. In order to again reduce uncertainties, we did not correct for the time dilatation effect by interpolating the flux (as in Lamb \& Reichart 2000); instead, we computed the time of the measurement in the burst rest-frame. This provides a better correction for the time dilatation.

Finally, we restricted the light curves to the $2.0-10.0 \mathrm{keV}$ $\mathrm{X}$-ray band, where the absorption is negligible. This allowed us to get rid of any other corrections for absorption by the ISM. We point out that the spectral analysis is done with a larger $\mathrm{X}$-ray band in order to reduce the spectral index error.

\section{Results}

\subsection{Results and statistical significance of the groups}

The result of this comparison is shown in Fig. 1. A zoomin on the clustered data is presented in Fig. 2. We first note that the two groups noted in Paper I are still present. The sample has now been extended to 17 bursts. All but one lie in one of the two groups. The only (notable) exception is GRB 980425; however the overall properties of this burst, associated with SN 1998bw are very peculiar compared to other GRBs (e.g. Galama et al. 1999b).

We now examine now the statistical significance of these two groups. In the following we call group I the set of GRB afterglows with the brightest luminosity, and group II the dimmer ones. There are six afterglows in group I, and ten in group II. One day after the burst, their mean fluxes are respectively $\sim(5 \pm 2) \times 10^{-12}$ and $\sim\left(3.5_{-2.7}^{+3.5}\right) \times 10^{-13} \mathrm{erg} \mathrm{cm}^{-2} \mathrm{~s}^{-1}$. We tested

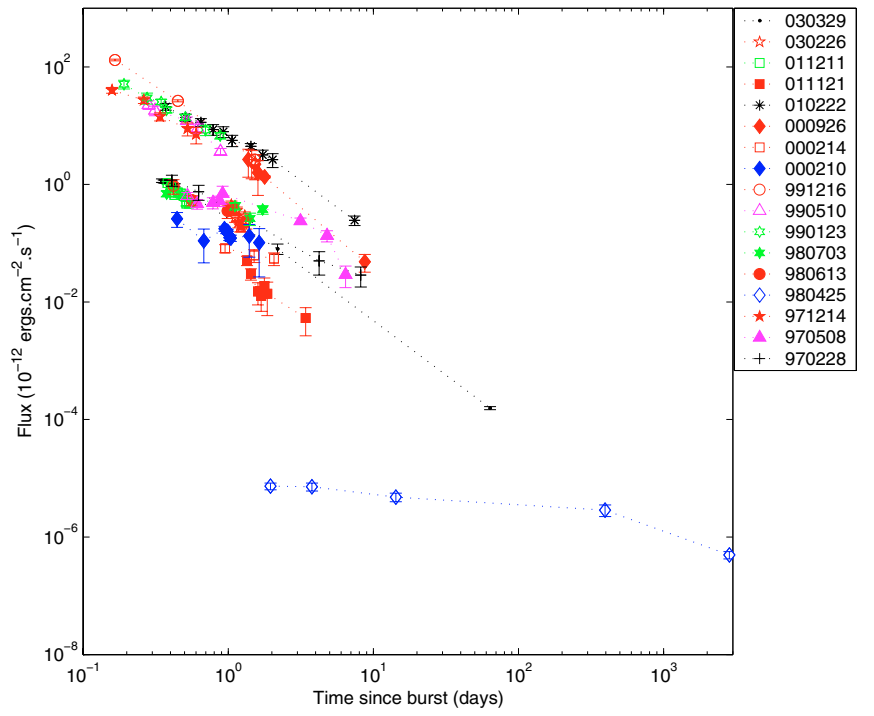

Fig. 1. X-ray light curves of the GRB afterglows rescaled at a common redshift of $z=1$. See the electronic version for a color version.

the hypothesis that a uniform luminosity distribution causes the observed distribution. The probability of getting the observed clustering by chance is only $1.64 \times 10^{-8}$. We also computed the probability of getting the observed diagram assuming a power law luminosity distribution, letting the index be a free parameter: the maximum probability is $1.10 \times 10^{-4}$ for an index value of -1 . We conclude that the observed clustering in two groups of the actual distribution is significant at at least to the $4 \sigma$ level.

\section{2. properties of the groups}

We first compute the mean spectral indices of these two groups: we find $\alpha=1.0 \pm 0.1$ and $\alpha=1.2 \pm 0.2$ for group $I$ and group II respectively. We note that the spectral indices are not statistically different. Since in paper I we indicated that the decay indices of these two groups were not compatible, we computed the mean decay index of the groups. We find $\delta=1.6 \pm$ 0.2 for group I. If we take into account all bursts of group II, we find $\delta=1.5 \pm 0.9$. However, if we tak into account only the bursts with a good decay constraint (hence ignoring GRBs with large error bars, i.e. GRB 011121, $\delta=4_{-2}^{+3}$, and GRB 030226, $\delta=2.7 \pm 1.6$ ), we get $\delta=1.1 \pm 0.2$ (note that we get $\delta=1.2 \pm$ 0.2 if we ignore only GRB 011121), a result compatible with Paper I. In order to test the statistical significance of the difference between the decay indices, we computed the probability that the observed repartition is due to only one population of GRBs. Using a Kolmogorov-Smirnov test we find that the probability is 0.13 , indicating that this distribution of decay indices may be due to only one population.

Figure 3 displays the distribution of decay indices versus the normalized flux at several times. In order to avoid a bias in this repartition, we interpolated the flux value using the two nearest data points, when known. This implies that there should be a good time coverage to have meaningful repartition. We made this computation at $0.6,0.9$ and 1.2 days. One can see in Fig. 3 the two groups, separated by one order of magnitude at 0.6 days. 


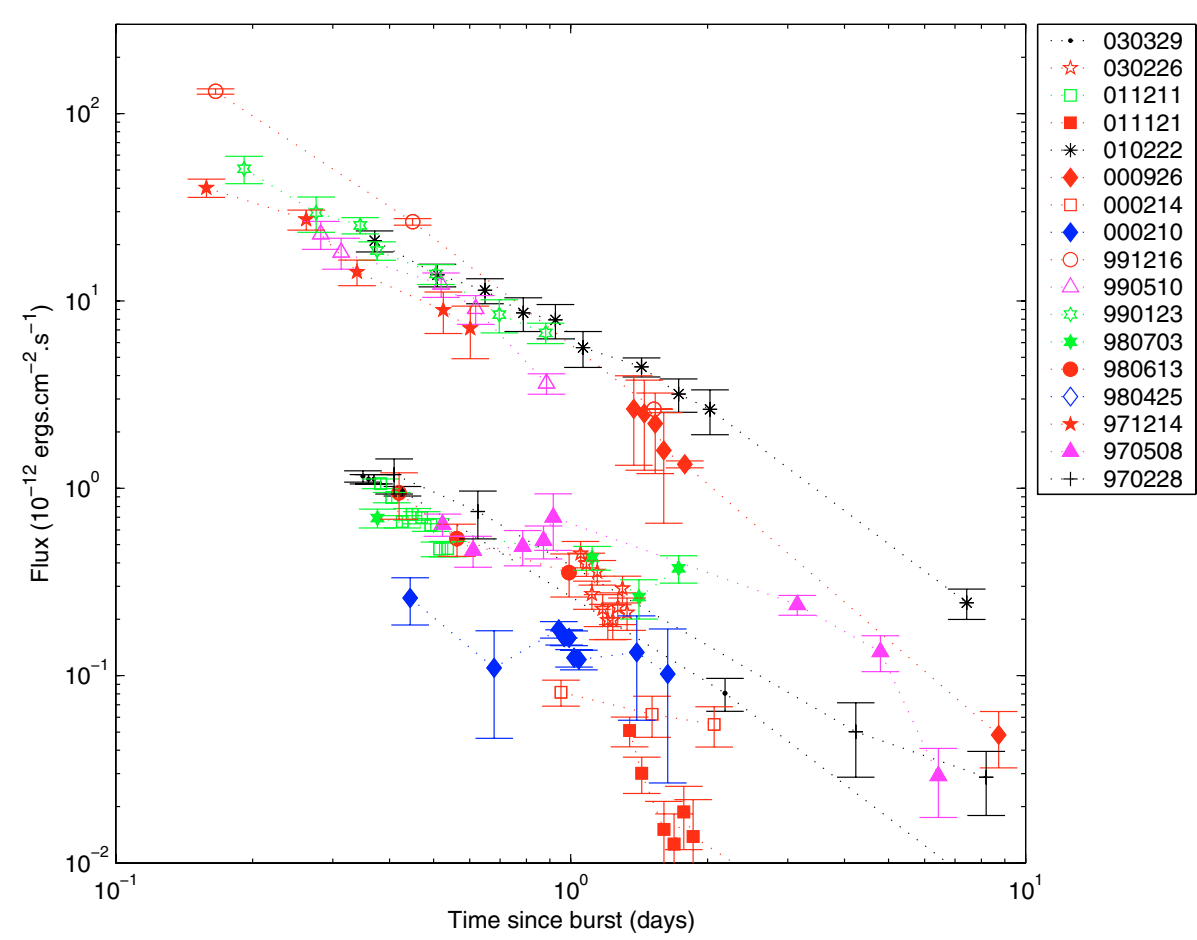

Fig. 2. X-ray light curves of the GRB afterglows rescaled to a common redshift $z=1$. We rescaled the previous figure in order to display the first 10 days of the afterglows. See the electronic version for a color version.
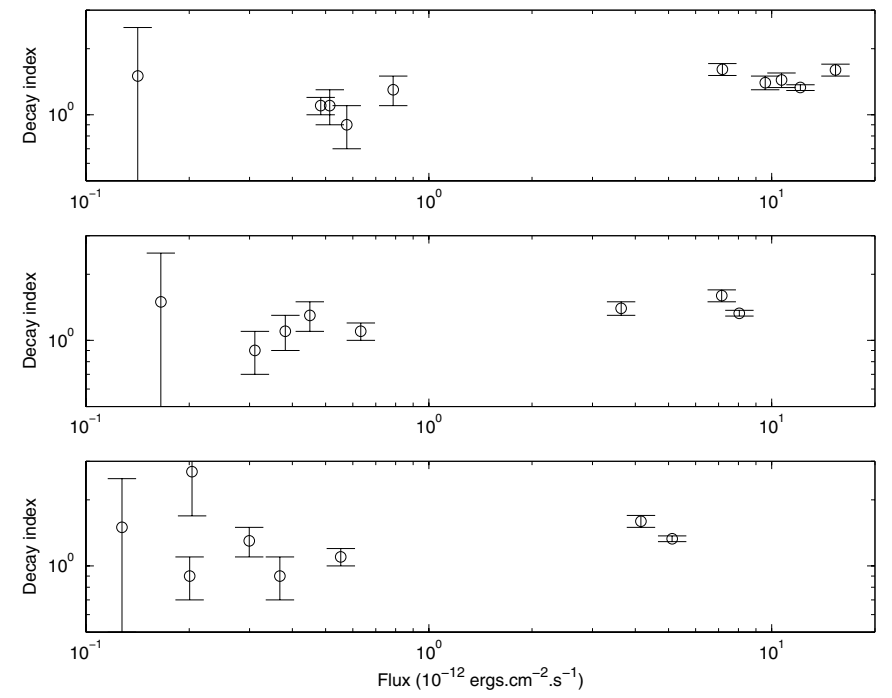

Fig. 3. X-ray decay indices versus the X-ray flux for several GRBs. The fluxes are taken at 0.6 (top panel), 0.9 (middle panel) and 1.2 (bottom panel) days.

\section{Discussion}

In Paper I we tried to explain the nature of these two groups in the framework of the fireball model using the adiabatic/radiative cooling of the fireball. With a value of $p$, the electron power law index, of 2.3, one should observe decay values of 1.68 and 1.22 for groups $I$ and $I I$ respectively (Sari et al. 1998): this is compatible with the observed values. However, one expects that when the cooling frequency of the fireball is equal to $v_{\mathrm{m}}$, the radiative fireball becomes adiabatic (Piran 1999). This would appear in the light curve as a flattening at that time (Sari et al. 1998). This is not the case in Fig. 1: none of the GRBs in group I displays such a feature. We thus cannot explain the nature of these groups by adiabatic/radiative cooling of the fireball.

We used the mean decay index of group I to interpolate the flux of GRB 971214 at large times (see Fig. 4). This interpolation seems to define a limit, where there is no data point belonging to group II. Most of all, some afterglow light curves of group II (GRB 970508, GRB 030329), which should cross this line, display a steepening. This is also true for the very faint part of this figure: GRB 980425 features a steepening.

One may argue that this limit is not valid, since some bursts of group I lie above the limit. We therefore computed then a new limit, using the parameters from the brightest burst (see Fig. 4). In this case we cannot draw any firm conclusion about the steepening. We investigate the possibility that this limit is due to instrumental effects: most of the afterglows are observed during the first 10 days of their decay. The only exceptions are GRB 980425 and GRB 030329 which are very unusual afterglows. We thus cannot use them to support any model. We conclude that this limit is valid at least for the first ten days of the afterglow.

In order to explain the observed GRB afterglow properties, we first assume that the two groups are not linked together. We use the relationships given in Zhang \& Meszaros (2004). In this framework we suppose that the afterglows from group I are produced by a constant density wind from the progenitor, and that afterglows from group II are expanding in an ISM of constant density. If we apply the results from Zhang \& Meszaros (2004), the value of the spectral index should be $(p-1) / 2 \sim 0.7$, different from what we observe for group $I$. 


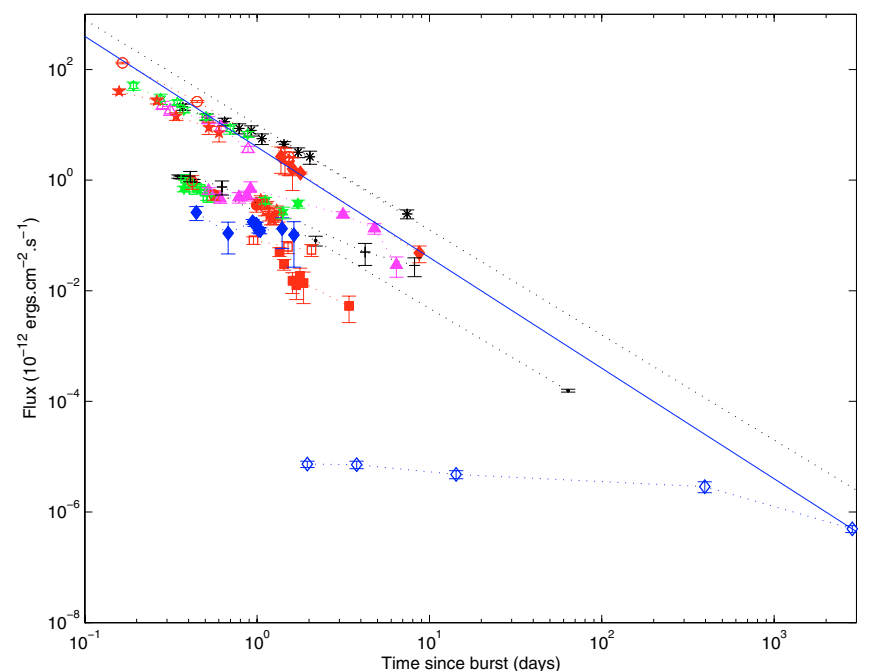

Fig. 4. X-ray light curve of the sample of GRB rescaled to a common distance corresponding to a redshift of $z=1$. We plotted the limiting fluxes computed as the mean of the bursts from group I (solid line) or as the mean power law corresponding to the brightest burst (dashed line).

Another possible explanation could be a difference in the value of $p$ of the two groups. In this case GRBs from group I should have $p=3.13$ and those from group II should have $p=2.13$, in order to explain all the X-ray data. However, we know from the broad band spectrum fitting of GRB 990123 that $p=2.28 \pm 0.05$ for this burst (Panaitescu \& Kumar 2002), not compatible with this hypothesis.

In the following we suppose that the two groups are linked together. In this case we should observe a break in the low group, otherwise the above mentioned limit would be crossed. Achromatic steepening (also called breaks) in light curves has been associated with jet-like features (see e.g. Tiengo et al. 2003): an increase in the opening angle of the jet implies a steepening in the afterglow light curve (Sari et al. 1999). We may suppose that the clustering in two groups is due to geometrical effects, such as beaming. Bursts from group I may have a smaller beaming factor than bursts from group II.

Berger et al. (2003) found evidence for a beaming effect on afterglow light curves. We report in Table 1 the beaming angle they derived for our bursts. We clearly see a trend: the bright afterglows have small beaming angles, as expected in this hypothesis. Note that this indicates that the fluxes we report here cannot be considered to be proportional to the luminosity. The latter quantity should be obtained taking into account the beaming factor and the burst parameters (temporal decay and spectral index). We can then explain the high decay index of group I by a small beaming angle. Group II is not affected at early times because of a larger beaming angle. However, in that hypothesis the decay index is not compatible with the value expected from the jet model ( 2.3, Sari et al. 1999). In addition, we do not expect such a clustering in two well separated groups from the distribution of GRB beaming angles.

Another possible interpretation can be given in the framework of the wind model. In this model, the cooling frequency increases with time (Chevalier \& Li 2000). As indicated in
Chevalier \& Li (2000) the light curve displays a steepening when the cooling frequency crosses the observed frequency: The effect is an increase of the decay index of 0.25 . Below the cooling frequency, the decay index is expected to be $\sim 1.6$, compatible with group $I$. The index difference between the two groups is $0.5 \pm 0.4$, compatible with the model value of 0.25 . In this model, if a segregation in decay indices versus time is expected from different GRBs, connected with the time at which the cooling frequency crosses the X-ray band, there is no particular reason for this to occur at a fixed time for several bursts.

As noted by Chevalier et al. (2004) some afterglow data can be fitted by either a wind model or an ISM model. On the other hand, Panaitescu \& Kumar (2001a, 2002) reported that the optical afterglow data cannot be fitted by a wind model for several bursts (e.g. GRB 990123).

If the GRB source is a very massive star, we expect that the progenitor will be surrounded by a medium with a wind profile (Chevalier \& Li 1999). Some refinements have been made to this model later in order to take into account a region where the wind profile ends in the ISM (Ramirez-Ruiz et al. 2001; Chevalier et al. 2004) with a termination shock. If this is the case, the fireball starts its expansion into a wind profile (the cooling frequency increases with time) then reaches a constant density medium, where the cooling frequency decreases as a function of time. We can model the light curve as follow (assuming $p=2.6$ ):

- first, the cooling frequency is below the X-ray frequency, and the flux decay index is -1.45 ;

- second, the cooling frequency crosses the X-ray band, the light curve displays a steepening and the decay index is -1.7 ;

- third, the afterglow reaches the termination shock and displays a flattening: the decay index becomes -1.2 ;

- last, the cooling frequency, which decreases with time as in the ISM model, again crosses the X-ray band, the light curve displays a steepening and the decay index becomes -1.45 .

The first break is expected to occur within hours (or less) after the burst, hence it is not observable in our sample. GRB afterglows from group II can be explained in this framework, since we we observe only the third and last segment mentioned above. Let us suppose now that the cooling frequency does not cross the X-ray band before the afterglow reaches the termination shock. In this case the first and the last segments only will be present, i.e., the light curve will decay as $t^{-1.45}$. This can explain the afterglow light curve of bursts from group I, i.e. they are observed before or after the termination shock. If this explanation is valid for the decay index values, no constraints are made on the burst brightness.

In this framework we also expect a variation of the spectral indices between the different segments (Zhang \& Meszaros 2004). The spectral index variation should be 0.5 . As we observe a variation of $0.2 \pm 0.3$, no firm conclusion can be derived.

Whatever the nature of these groups, we expect each model parameter to vary within a certain range. Hence the flux should 
vary within two extrema, while we observe clustering around two groups. Variations from this clustering are only observed for two bursts and they can be explained by unusual properties:

- GRB 011121 deviates from the other bursts of group II by a high decay index. This can be explained if one notes that the optical light curve of GRB 011121 displayed an achromatic break before the X-ray observation, which may be attributed to a jet feature (Greiner et al. 2003).

- The case of GRB 000214 is due to the uncertainty in the redshift. All the figures where made with a value of 0.42 , but the redshift is badly constrained, and could have any value between 0.37 and 0.47 .

The clustering may be an indication that GRB physical parameters (the microphysics parameters, the energy and the beaming angle) and their environmental parameters (density, profile) should lie within a very narrow range, and might even be identical from burst to burst.

\section{Conclusions}

We analyzed the X-ray afterglow data of seventeen GRBs with known redshift values. Our main conclusions are:

- We confirm our result from Paper I (Boër \& Gendre 2000) that GRB X-ray afterglows can be segregated by their decay index and brightness. The interpretation of this clustering within the framework of the standard fireball model remains unclear.

- We report evidence for a flux limit, i.e. all burst display a flux below $9 \times 10^{-12} \mathrm{erg} \mathrm{s}^{-1} \mathrm{~cm}^{-2}$ one day after the burst.

Long lasting (1 month or more), and continuous X-ray observations of GRB are needed to confirm the validity of the above reported limit after ten days. The decaying nature of GRB afterglows and their faintness at those times requires the use of high-sensitivity facilities, such as the XMM-Newton observatory.

We note that it is certainly desirable to increase the statistics, and hence the size of the sample. This makes it necessary to detect more X-ray and optical afterglows and to determine their redshift, as it will be possible after the launch of the SWIFT experiment. Finally, we note that infrared observations of GRB afterglows, since they are weakly affected by interstellar absorption, may lead to results comparable with those reported here.

Acknowledgements. We acknowledge the use of the web page of J. Greiner (http://www.mpe.mpg.de/ jcg/grbgen.html). We thank L. Piro, M. DePasquale and A. Galli for useful discussions, comments, and for allowing us to use unpublished data. This work was supported by the EU FP5 RTN 'Gamma ray bursts: an enigma and a tool'. We finally thank P. Meszaros for his comments.

\section{References}

Akerlof, C., Balsano, R., Barthelmy, S., et al. 2000, ApJ, 532, L25

Arnaud, K. A. 1996, in Astronomical Data Analysis Software and Systems V, ed. G. Jacoby, \& J. Barnes, ASP Conf. Ser., 101, 17
Berger, E., Kulkarni, S. R., \& Frail, D. A. 2003, ApJ, 590, 379

Boër, M., Bringer, M., Klotz, A., et al. A\&AS, 138, 579

Boër, M., \& Gendre, B. 2000, A\&A, 361, L21

Chevalier, R. A., \& Li, Z. Y. 1999, ApJ, 520, L29

Chevalier, R. A., \& Li, Z. Y. 2000, ApJ, 536, 195

Chevalier, R. A., Li, Z. Y., \& Fransson, C. 2004, ApJ, 606, 369

Costa, E., Frontera, F., Heise, J., et al. 1997, Nature, 387, 783

Costa, E. 1999, A\&AS, 138, 425

Dai, Z. G., \& Lu, T. 1998, MNRAS, 298, 87

Dezalay, J. P., Lestrade, J. P., Barat, C., et al. 1996, ApJ, 471, L27

Diercks, A., Deutsch, E. W., Castander, F. J., et al. 1998, ApJ, 503, L105

Djorgovski, S. G., Kulkarni, S. R., Odewahn, S. C., \& Ebeling, H. 1998, GCN 117

Frail, D. A., Berger, E., Galama, T., et al. 2000, ApJ, 538, L129

Frail, D. A., Kulkarni, S. R., Sari, R., et al. 2001, ApJ, 562, L55

Galama, T., Groot, P. J., van Paradijs, J., et al. 1997, Nature, 387, 479

Galama, T., Briggs, M. S., Wijers, R. A. M., et al. 1999a, Nature, 398, 394

Galama, T., Vreeswijk, P. M., van Paradijs, J., et al. 1999b, A\&AS, 138,465

Gendre, B., Barret, D., \& Webb, N. A. 2003, A\&A, 400, 521

Greiner, J., Klose, S., Salvato, M., et al. 2003, ApJ, 599, 1223

Halpern, J. P., Uglesich, R., Mirabal, N., et al. 2000, ApJ, 543, 697

Klebesadel, R. W., Strong, I. B., \& Olson, R. A. 1973, ApJ, 182, L85

Kouveliotou, C., Meegan, C. A., Fishman, G. J., et al. 1993, ApJ, 413, L101

Kuulkers, E., Antonelli, L. A., Kuiper, L., et al. 2000, ApJ, 538, 638

Lamb, D. Q., \& Reichart, D. E. 2000, ApJ, 536, L1

Meszaros, P., \& Rees, M. J. 1997, ApJ, 476, 232

Meszaros, P., Rees, M. J., \& Wijers, R. A. M. J. 1998, ApJ, 499, 301

Meszaros, P. 2001, Science, 291, 79

Metzger, M. R., Djorgovski, S. G., Kulkarni, S. R., et al. 1997, Nature, 387,879

Panaitescu, A., Meszaros, P., \& Rees, M. J. 1998, ApJ, 503, 314

Panaitescu, A., \& Kumar, P. 2001a, ApJ, 554, 667

Panaitescu, A., \& Kumar, P. 2001b, ApJ, 560, L49

Panaitescu, A., \& Kumar, P. 2002, ApJ, 571, 779

De Pasquale, M., et al. 2004, private communication

Pedersen, H., Jaunsen, A. O., Grav, T., et al. 1998, ApJ, 496, 311

Penn, U. L. 1999, ApJS, 120, 49

Pian, E., Amati, L., Antonelli, L. A., et al. 2000, ApJ, 536, 778

Pian, E., Soffita, P., Alessi, A., et al. 2001, A\&A, 372, 456

Piran, T. 1999, Physics Reports, 314, 575

Piro, L., Amati, L., Antonelli, L. A., et al. 1998, A\&A, 331, L41

Piro, L., Costa, E., Feroci, M., et al. 1999, A\&AS, 138, 431

Piro, L., Garmire, G., Garcia, M., et al. 2000, Science, 290, 955

Price, P. A., Kulkarni, S. R., Berger, E., et al. 2003, ApJ, 589, 838

Ramirez-Ruiz, E., Dray, L. M., Madau, P., \& Tout, C. A. 2001, MNRAS, 327, 829

Rees, M. J., \& Meszaros, P. 1992, MNRAS, 258, 41

Reeves, J. N., Watson, D., Osborne, J. P., et al. 2001, Nature, 416, 512

Reichart, D. E. 1999, ApJ, 521, L111

Rhoads, J. E. 1997, ApJ, 487, L1

Sari, R., Piran, T., \& Narayan, N. 1998, ApJ, 497, L17

Sari, R., Piran, T., \& Halpern, J. P. 1999, ApJ, 519, L17

Stanek, K. Z., Garnavich, P. M., Kaluzny, J., Pych, W., \& Thompson, I. 1999, ApJ, 522, L39

Tiengo, A., Mereghetti, S., Ghisellini, G., et al. 2003, A\&A, 409, 983

Vreeswijk, P. M., Galama, T. J., Owens, A. N., et al. 1999, A\&AS, 138,447

Zhang, B., \& Meszaros, P. 2004, Intern. J. Mod. Phys. A, in press [arXiv:astro/ph-0311321] 